\title{
El programa común complementario
}

\author{
Josep $\mathrm{M}^{\mathrm{a}}$ Fornells, $\mathrm{M}^{\mathrm{a}}$ Antonia Sans, $\mathrm{M}^{\mathrm{a}}$ Josep Cerqueira, Salvador Hernández \\ Red de Comisiones de Docencia y Asesoras de Cataluña
}

\section{JUSTIFICACIÓN}

La formación formal de los actuales especialistas tiene unos déficits en sus contenidos que vienen determinados por no tener en cuenta como debe ser y que debe saber el médico que necesita la sociedad del siglo XXI. Así, nos encontramos con déficits importantes en aspectos humanísticos, éticos, técnicas de comunicación, investigación médica y la gestión clínica.

Todos estos aspectos formativos aun no siendo elementos técnicos, no dejan de ser importantes para la formación de buenos especialistas desde una visión más amplia. Los argumentos para la incorporación de estas materias en la formación de especialistas los encontramos en los siguientes hechos:

1.- Cambios en el rol médico-paciente de los que se deriva que se primó, en primer lugar, la defensa de los derechos y la autonomía del paciente (carta de derechos y deberes del enfermo), y por otro lado el aumento del nivel cultural de la población al tener ésta mayor acceso y comprensión de la información médica.

2.- Cambios demográficos que comportan cierto desconocimiento de la realidad social de la población inmigrada (cultura, idioma, religión, etc..) Este hecho hace más vulnerable al especialista y se ponen más en evidencia los déficits en habilidades comunicativas.

3.- La ley de ordenación de las profesiones sanitarias, que en su articulo 5 apartado 1,c resalta el deber del profesional de respetar, la personalidad dignidad e intimidad de las personas a su cuidado y deben respetar la participación de los mismos en las tomas de decisiones que les afecten. En todo caso deben ofrecer una información adecuada y suficiente para que aquéllos puedan ejercer su derecho sobre el consentimiento a dichas decisiones.

En el apartado 1,b del mismo artículo hace referencia al deber que tiene el profesional de hacer un uso racional de los recursos diagnósticos y terapéuticos. En definitiva ha de conocer la dimensión económica de la infra o supra utilización o de la utilización inadecuada de los recursos que se tienen al alcance.

4.- La opinión de los propios profesionales sobre la formación recibida en aspectos que consideran de máxima relevancia. (1)

\section{ANTECEDENTES}

El plenario de las Comisiones de Docencia y
Asesoras de Cataluña debatió en 1995 la necesidad de formación de los residentes en ámbitos genéricos que no estaban contemplados en los programes establecidos por las correspondientes Comisiones Nacionales de la especialidad. Nacía así el Programa Común Complementario (PCC), que se inicio en 1996 con un plan piloto en cuatro centros hospitalarios y dos Unidades docentes de Medicina Familiar y Comunitaria. Con posterioridad otros centros y unidades docentes adoptaron el PCC.

El nombre mismo del programa nos señala las dos características básicas del mismo. Por una parte es común a los residentes de todas les especialidades y está pensado para que sea válido para todas ellas; ya sean médicas, quirúrgicas o las que corresponden a servicios centrales. Por otra parte es complementario al programa específico oficial de cada especialidad con el objetivo de proporcionar una formación más integral, que facilite al futuro especialista una mejor respuesta a nuevas necesidades.

Los centros continúan teniendo total autonomía para organizar las actividades formativas que crean más oportunas. El PCC no pretende más que dar una formación de mínimos, de calidad para todos los residentes de Cataluña.

\section{ESTRUCTURA}

El PCC se estructuró en cinco módulos formativos distribuidos en tres años con un total de 154 horas, sin contar los trabajos individuales tutorizados. Las materias que se priorizaron fueron las siguientes:

Ir año de residencia:

- Metodología científica I

20 horas

Introducción a la investigación

- Habilidades comunicativas

- Resucitación cardiopulmonar

$2 n$ año de residencia:

- Metodología Científica II

Bioestadística

- Bioética

30 horas

4 horas

20 horas

30 horas

$3 r$ año de residencia:

- Metodología científica III

20 horas

Comunicación científica

- Gestión asistencial

30 horas

Se puso especial énfasis en los aspectos metodológicos ya que se quería evitar una formación excesivamente teórica y por contra se intentó un aprendizaje 
que estuviera cerca del modelo de formación basado en la experiencia que ya seguían los residentes.

\section{PUESTA EN MARCHA}

El Instituto de Estudios de la Salud se responsabilizó de acreditar a los centros que querían poner en marcha el PCC valorando el programa presentado por los centros y haciendo el seguimiento de los mismos.

La presentación del programa antes mencionado debía incluir los siguientes apartados para cada modulo.

- Objetivos de aprendizaje

- Contenidos

- Metodología

- Evaluación de los residentes

- Relación de profesores con un breve currículum

\section{VALORACIÓN DEL PCC}

La implementación del PCC tuvo un alcance limitado puesto que fue adoptado de manera minoritaria por los centros acreditados para la formación especializada. No obstante sirvió para poner en el orden del día la necesidad de formación en materias transversales y muchos centros si bien no adoptaron el PCC en su integridad sí que pusieron en marcha varios de los módulos que configuraban el PCC. Aparte tuvo un carácter pionero en el resto del estado siendo el precursor de experiencias similares desarrolladas en otras comunidades autónomas.

En el apartado de aspectos a mejorar habría de señalar las resistencias que se han observado por parte de ciertos sectores del staff y de algunos jefes de servicio y que en ocasiones han actuado no favoreciendo la participación de los residentes en el PCC. En parte se puede explicar por problemas de "comunicación" tanto vertical como horizontal y por un conflicto de "modelos".

Una critica generalizada de los residentes es la falta de compatibilidad entre el PCC y su actividad asistencial así como los horarios, total o parcialmente extralaborales.

\section{PERSPECTIVAS DE FUTURO}

La Red de Comisiones de Docencia y Asesoras de Cataluña encargó a un grupo de trabajo la elaboración de una propuesta para la dinamización del PCC. A falta de la presentación formal de las recomendaciones de este Grupo y de su aprobación por la Comisión Permanente de la Red podemos avan- zar lo que serian conclusiones preliminares y que constituirán los ejes de su posterior desarrollo.

\section{ESTRUCTURA DEL PCC}

- Se define una nueva estructura del PCC que pasa de las 154 horas iniciales a 85 horas a desarrollar en 2 años:

- Habilidades comunicativas 20 horas

- Bioética

20 horas

- Lectura critica de artículos

y búsqueda bibliográfica

25 horas

- Salud Publica

10 horas

- Gestión Clínica

10 horas

- La reducción global de horas tiene que enmarcarse en una estrategia de mínimos para facilitar su cumplimiento en todos los centros acreditados. Por otra parte la reducción más significativa corresponde al modulo de metodología científica para el que se considera que existe suficiente oferta en nuestro medio.

\section{PUESTA EN MARCHA Y DESARROLLO}

- Se consideran plenamente vigentes los principios que inspiraron la creación del PCC hace ya 10 años.

- La puesta en marcha del PCC debe seguir criterios de flexibilidad y descentralización. Se favorecerá una política de formación de formadores para permitir la máxima autonomía posible por parte de los centros en la formación de sus residentes en temas transversales.

- Deben determinarse los criterios de acreditación de los programas así como los de evaluación de los residentes, con el objetivo de garantizar una adecuada calidad.

- El PCC pretende marcar unos objetivos mínimos y en ningún caso debe ser un obstáculo para centros que quieran plantearse objetivos de formación más ambiciosos.

- La realización del PCC por parte de los residentes debe tener una valoración trascendente que estimule su realización. Se debe contemplar su valoración curricular.

- Se debe contemplar el sistema de financiación del mismo.

- Los centros con pocos residentes deberán establecer alianzas con otros centros de su área geográfica. El Instituto de Estudios de la Salud facilitará la organización a demanda de los diferentes módulos. 